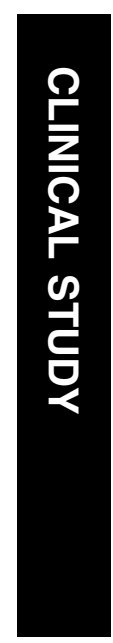

\section{Relationship between GDx VCC and Stratus OCT in juvenile glaucoma}

\begin{abstract}
Purpose To compare the ability of scanning laser polarimetry (GDx VCC) and optical coherence tomography (OCT) to discriminate eyes with juvenile glaucoma from normal eyes and to assess the relationship between their parameters.

Methods A total of 24 glaucomatous eyes of 24
\end{abstract} patients and 24 normal eyes were enrolled. The age range of the patient was 11-40 years with a mean age of $25.1 \pm 8.2$ years. Contol groups consisted 24 eyes of 24 individuals without glaucoma with a mean age of $33.2 \pm 8.2$ years. All subjects underwent a full ophthalmic examination, automated perimetry, GDx VCC, and OCT. Correlation coefficients between the

Department of Ophthalmology, Farabi Eye Hospital, Farabi Eye Research Center, Tehran University of Medical Sciences, Tehran, Iran

Correspondence:

S Moghimi, Department of Ophthalmology,

Farabi Eye Hospital, Eye Research Center, Tehran University of Medical Sciences,

Quazvin Sq.,

Tehran 1336616351, Iran

Tel: + 982155416134;

Fax: +982155416134 .

E-mail: sasanimii@

yahoo.com

Received: 22 December 2008

Accepted in revised form:

3 January 2009

Published online:

20 February 2009

The authors state that they have only academic interest and no financial interest in this study parameters of OCT and GDx VCC were

calculated. We calculated the area under the receiver operating characteristic curve (AROC) for the main parameters of GDx VCC and OCT. Results Statistically significant correlations were observed between GDx VCC and OCT parameters. Pearson coefficients ranged from 0.75 for inferior average to 0.86 for nerve fibre indicator (NFI)/average thickness OCT. The greatest AROC parameter in OCT (inferior average: 0.92) had a lower area than that in GDx VCC (NFI: 0.99). There was a significant statistical significance in all visual field, GDx VCC, and OCT variables between two groups $(P<0.05)$.

Conclusions Many GDx VCC parameters were significantly correlated with those of the OCT in patients with juvenile glaucoma. Inferior average and NFI had the greatest AROC parameter in OCT and GDx VCC, respectively. NFI had high sensitivity and specificity for the diagnosis of JOAG.

Eye (2009) 23, 2182-2186; doi:10.1038/eye.2009.15; published online 20 February 2009

Keywords: juvenile glaucoma; optical coherence tomography; scanning laser polarimetry
R Zareii, M Soleimani, S Moghimi, Y Eslami, G Fakhraie and H Amini

Introduction

Glaucoma is an optic neuropathy with the loss of retinal ganglion cells and this loss clinically is defined by some thinning of the retinal nerve fibre layer (RNFL). ${ }^{1}$ Recently, several studies have designed to report the relationship between RNFL damage and visual function in glaucomatous optic neuropathy. ${ }^{1-3}$

Optical coherence tomography (OCT) and scanning laser polarimetry (SLP) are two techniques that measure peripapillary RNFL thickness. OCT can obtain a cross section of the retina with interferometry based on the reflectivity of the different layers of the retina. GDx VCC, the latest version of SLP, is based on a variable corneal compensation and estimates the thickness of RNFL by measuring the sum of retardation from forming ganglion cell axons. ${ }^{2-4}$ Earlier trials showed high accuracy of both methods in detection of glaucoma, ${ }^{5-7}$ but there is no study to define the correlation between these two measurements in juvenile open angle glaucoma (JOAG). The purpose of this study was to investigate the relationship between Stratus OCT and GDx VCC in juvenile glaucoma and to determine the parameters with the best diagnostic performance for each instrument.

\section{Materials and methods}

A total of 24 glaucomatous eyes of 24 patients meeting the inclusion criteria were included in this study. Control group consists of 24 eyes of 24 patients that referred from other clinics. Informed consent was obtained from all patients included in this cross-sectional study. Only one eye of each patient was included in the trial. Patients were defined as affected by JOAG when they showed at least two of the following criteria: glaucomatous visual field (VF) defects (based on the pattern standard deviation (PSD) and the mean deviation (MD)), 
ocular hypertension (IOP $>21 \mathrm{mmHg}$ ), and optic nerve head glaucomatous changes (disc asymmetry between the eyes that was $\geqslant 0.2$, rim thinning, notching, excavation, or RNFL defect). Classification of JOAG was based on the age of diagnosis, ranging between the age of 10 and 40 years.

Inclusion criteria were BCVA $>0.5$, open anterior chamber angle, absence of other ocular pathologies, reliable SAP, and good SLP and OCT image quality. Exclusion criteria included refractive error higher than $5 \mathrm{D}$ sphere and 3D cylinder; pupils $<3 \mathrm{~mm}$ in diameter; presence of secondary causes of glaucoma, diabetes mellitus, neurological disorders, earlier ocular surgery, or medication that might alter SAP results.

All subjects underwent a complete ophthalmic examination. Static automated perimetry (Humphrey field analyzer (HFA) II, central 24-2 SITA FAST strategy; Carl Zeiss Meditec, Dublin, CA, USA), GDx VCC imaging, and Stratus OCT imaging were performed. Control patients had IOP $<22$ and normal VF.

\section{OCT}

After dialation of pupil, OCT imaging (Stratus OCT, software 3.0; Carl Zeiss Meditec) of the peripapillary RNFL was performed using high resolution scans with a diameter of $3.4 \mathrm{~mm}$ centred on the optic disc (fast RNFL scan). Average thickness, superior and inferior averages were obtained. Mean of three measurements was used for the analysis.

\section{GDx}

GDx VCC imaging (Laser Diagnostic Technology, San Diego, CA, USA) was performed using a scan circle of $3.2 \mathrm{~mm}$ diameter centred on the optic disc, and the mean of three measurements was used again. All images with quality score gradings $<8$ were excluded. TSNIT average, nerve fibre indicator (NFI), and superior and inferior averages were obtained.

\section{Statistical analysis}

Data were analysed by SPSS 13 (SPSS Inc., Chicago, IL, USA). T-test and $\chi^{2}$-test were used for the quantitative and qualitative data, respectively. Pearson's correlation coefficient $(r)$ was calculated for each pair of parameters, and $P$-value of $\leqslant 0.05$ were considered statistically significant. The area under the receiver operating characteristic curve (AROC) and sensitivity at $90 \%$ specificity for each parameter were also calculated.

\section{Results}

A total of 24 glaucomatous eyes of 24 patients were included in this study (14 male and 10 female patients). The age range of patients was 11-40 years with a mean age of $25.1 \pm 8.2$ years. All glaucomatous eyes had VF loss of $-6.04 \pm 2.76 \mathrm{~dB}$ MD and 5.59 \pm 3.17 PSD.

Parameters of peripapillary RNFL using GDX VCC were measured as $41.20 \pm 5.43 \mu \mathrm{m}$ TSNIT average, $57.04 \pm 31.04 \mathrm{NFI}, 51.04 \pm 10.75 \mu \mathrm{m}$ superior average, and $44.31 \pm 8.29 \mu \mathrm{m}$ inferior average. In Stratus OCT, measured parameters were $77.80 \pm 19.32 \mu \mathrm{m}$ average thickness, $84.29 \pm 22.30 \mu \mathrm{m}$ superior average, and $72.29 \pm 19.23 \mu \mathrm{m}$ inferior average.

The relationships between GDx VCC and Stratus OCT parameters are represented in Figures 1-4. Statistically significant correlations were observed in each parameter $(P<0.005)$. Pearson coefficients were as follows: 0.80 for TSNIT average/average thickness OCT (Figure 1), -0.86 for NFI/average thickness OCT (Figure 2), 0.86 for superior average (Figure 3 ), and 0.75 for inferior average (Figure 4). All correlations were statistically significant in each parameter, and had positive correlations except for NFI.

A total of 24 normal eyes of 24 normal individual (12 male, 12 female subjects) were included in the study. Mean age in this group was $33.2 \pm 8.2$ years (range: 21-42). There was no significant difference between the two groups in age and sex. MD and PSD in normal group were $-0.50 \pm 0.56$ and $1.55 \pm 0.36$, respectively. GDx parameters in control group were $56.58 \pm 5.53$ for TSNIT average, $19.04 \pm 4.48$ for NFI, $64.58 \pm 6.16$ for superior average, and $65.16 \pm 6.50$ for inferior average. Average thickness, superior and inferior averages measured by OCT were $100.38 \pm 12.66,117.12 \pm 16.44$, and $120.00 \pm 13.01$, respectively. There was a significant statistical significance in all VF, GDx VCC, and OCT variables between two groups ( $P<0.05$, Student's $t$-test).

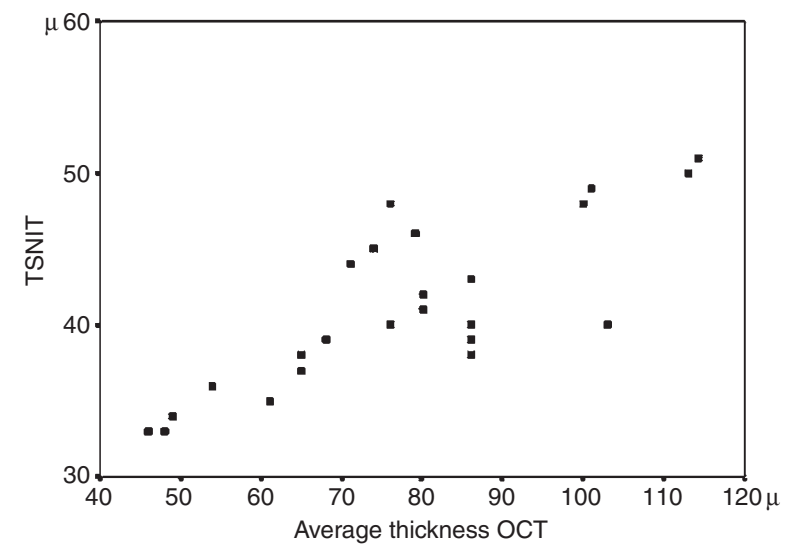

Figure 1 Correlation between TSNIT average of GDx and average thickness of OCT $(r=0.80)$. 


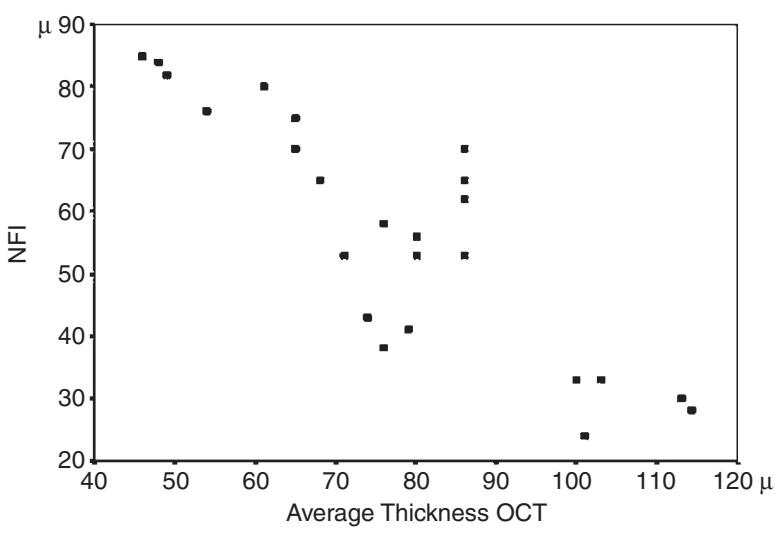

Figure 2 Correlation between NFI (nerve fibre indicator) of GDx and average thickness of OCT $(r=0.86)$.

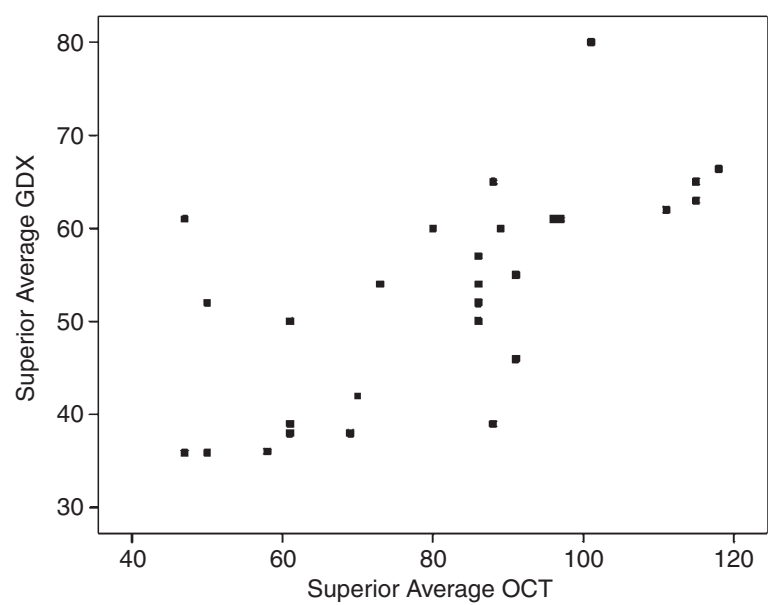

Figure 3 Correlation between superior averages of GDx and OCT $(r=0.86)$.

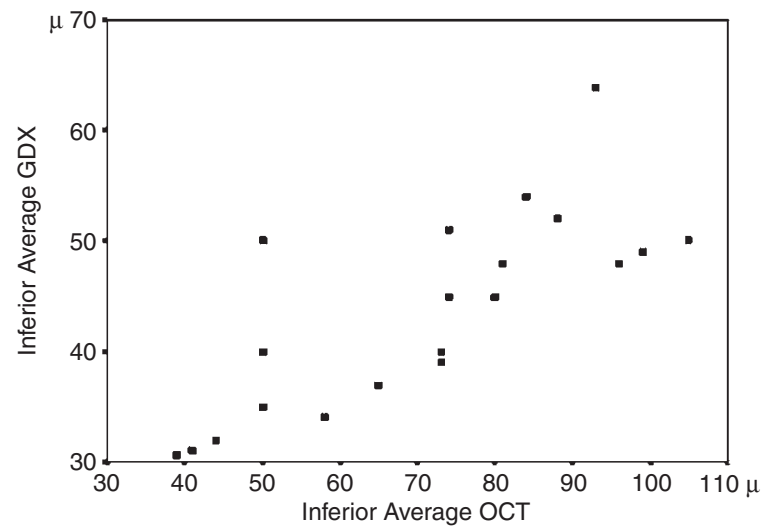

Figure 4 Correlation between inferior averages of GDx and OCT $(r=0.75)$

The greatest AROC parameter in OCT (inferior average: 0.92 ) had a lower area than that in GDx VCC (NFI: 0.99). The AROC for GDx parameters ranged from
Table 1 Sensitivity at $90 \%$ specificity and the AROC of main GDx and OCT parameters

\begin{tabular}{lccc}
\hline Test result variable(s) & Area & P-value & $\begin{array}{c}\text { Sensitivity at 90\% } \\
\text { specificity (\%) }\end{array}$ \\
\hline GDX parameters & & & \\
$\quad$ TSNIT average & 0.98 & 0.000 & 95.8 \\
$\quad$ Superior average & 0.85 & 0.000 & 58.3 \\
$\quad$ Inferior average & 0.92 & 0.000 & 91.7 \\
NFI & 0.99 & 0.008 & 95.8 \\
OCT parameters & & & \\
$\quad$ Average thickness & 0.83 & 0.000 & 41.7 \\
Superior average & 0.87 & 0.000 & 62.5 \\
$\quad$ Inferior average & 0.92 & 0.000 & 83.3 \\
\hline
\end{tabular}

0.85 to 0.99 , with greatest associated with NFI and TSNIT average ( 0.99 and 0.98 , respectively). The sensitivities at $90 \%$ specificity of the GDx parameters ranged from $58.3 \%$ of the superior average to $95.8 \%$ of NFI (Table 1). For OCT parameters, AROC ranged from 0.83 of average thickness to 0.92 of inferior average. The sensitivity at $90 \%$ specificity of the OCT parameters was greatest for inferior average (83.3\%; Table 1).

\section{Discussion}

Earlier studies have shown that structural damage of the optic nerve head and peripapillary RNFL precedes detectable VF loss using SAP; ${ }^{8} 40 \%$ axonal loss may occur before any detectable feature in SAP. ${ }^{9,10}$ However, conventional tools for the structural measurement in glaucoma, such as photography, are subjective and difficult to do; however, there is much variability between examiners. ${ }^{11,12}$ So, structural measurement techniques have been recently used to obtain objective quantitative data.

GDx and OCT allow quantitative and reproducible measurements of the $\mathrm{ONH}$ and the peripapillary RNFL. ${ }^{13,14}$ They can show evidence of RNFL loss up to 6 years before a detectable change in standard automated perimetry. ${ }^{8}$ It has been shown that both GDx and OCT have similar ability in the diagnosis of early-to moderate glaucoma. ${ }^{2,15}$ Both of them are able to provide reproducible measurements of RNFL thickness ${ }^{13,14}$ and to differentiate glaucomatous from normal eyes. ${ }^{16,17}$

There are some studies that concentrated on comparison between GDx VCC and OCT in glaucoma, ${ }^{2,18-21}$ but none of them focused on patients with juvenile glaucoma, who perhaps need more attention in society.

Total average RNFL thickness measured with Stratus OCT and GDx VCC were highly correlated, and those measurements of Stratus OCT and GDx VCC RNFL were 
significantly correlated in the study by Leung et $a l^{21}$. Chang and Sohn ${ }^{18}$ also reported that GDx VCC and Stratus OCT are highly correlated in glaucomatous eyes. In our study, in juvenile glaucoma patients, parameters of GDx VCC and Stratus OCT were significantly correlated with each other. Correlation coefficients were highest for NFI/average thickness OCT. TSNIT average/ average thickness OCT, superior and inferior averages were also well correlated. Higher correlations were also observed in NFI/average thickness OCT and superior average in earlier studies. ${ }^{18,22}$

NFI is a special retardation parameter in GDx-VCC that indicates the likelihood that glaucoma is present. It has a close relationship with RNFL thickness and VF status in glaucoma. ${ }^{23}$ Average thickness in OCT parameters had been shown to have the strongest correlation with the VF defect. ${ }^{22}$ So the strongest correlation between NFI and average thickness detected in several studies might be meaningful. Similar to earlier studies in $\mathrm{POAG}^{2,24}$ the best diagnostic performance for GDx VCC was the NFI, with an AROC of 0.99. Unlike earlier reports ${ }^{2,21}$ the sensitivity ( $95 \%$ at fixed specificity of $90 \%$ ) seems to be high for this variable in JOAG.

The first areas to be damaged in glaucoma seem to be in the inferotemporal and superotemporal areas. ${ }^{25}$ Evaluating RNFL thickness in the superior and inferior regions is often the best way of discriminating healthy eyes from eyes with early-to-moderate glaucoma, using OCT. Although some studies showed that superior average is the best OCT parameter for detecting glaucomatous eyes, ${ }^{2,26}$ in most other studies $5,16,17,26$ inferior average has the best diagnostic performance. Similar to these studies, in our patients, the largest AROC for OCT indices was inferior average. Our results show higher sensitivity (83\%), but nearly same specificity (89.4\%) for our best OCT parameter (inferior average) than most earlier studies. ${ }^{2,27}$

Brusini et $a l^{2}$ showed that the best performing indices for the GDx VCC and OCT gave similar AROCs. In our study, NFI of GDx has better discrimination than inferior average of OCT. It has higher sensitivity $(95 \%)$ than inferior average of OCT at fixed specificity of $90 \%$. Earlier studies show that OCT and GDx have moderate sensitivity with high specificity for the diagnosis of early glaucoma. ${ }^{2,27}$ Most of these studies were on early glaucoma that had small, localised RNFL thickness. One reason for our better results might be that we did not exclude patients with advanced juvenile glaucoma. So sensitivity of these tests was more in our study.

In conclusion, our results showed that parameters of GDx VCC and Stratus OCT are significantly correlated in juvenile glaucomatous patients. The best discriminating parameter in GDx (NFI) had higher AROC than the best parameter in OCT. NFI had high sensitivity and specificity for the diagnosis of JOAG.

\section{References}

1 Ma KT, Lee SH, Hong S, Park KS, Kim CY, Seong GJ et al. Relationship between the retinal thickness analyzer and the GDx VCC scanning OCT optical coherence tomograph, and Heidelberg retina tomograph II confocal scanning laser ophthalmoscopy. Korean J Ophthalmol 2008; 22: 10-17.

2 Brusini P, Maria L, Salvetat ML, Tosoni C, Parisi L, Felletti M. Comparison between GDx VCC scanning laser polarimetry and Stratus OCT optical coherence tomography in the diagnosis of chronic glaucoma. Acta Ophthalmol Scand 2006; 84: 650-655.

3 Izatt JA, Hee MR, Swanson EA, Lin CP, Huang D, Schuman JS et al. Micrometer-scale resolution imaging of the anterior eye in vivo with optical coherence tomography. Arch Ophthalmol 1994; 112: 1584-1589.

4 Huang XR, Knighton RW. Linear birefringence of the retinal nerve fiber layer measured in vitro with a multispectral imaging micropolarimeter. J Biomed Opt 2002; 7: 199-204.

5 Medeiros FA, Zangwill LM, Bowd C, Mohammadi K, Weinreb RN. Comparison of scanning laser polarimetry using variable corneal compensation and retinal nerve fiber layer photography for detection of glaucoma. Arch Ophthalmol 2004; 122: 698-704.

6 Medeiros FA, Zangwill LM, Bowd C, Vessani RM, Susanna Jr R, Weinreb RN. Evaluation of retinal nerve fiber layer, optic nerve head, and macular thickness measurements for glaucoma detection using optical coherence tomography. Am J Ophthalmol 2005; 139: 44-55.

7 Sheffield VC, Stone EM, Alward WLM, Drack AV, Johnson AT, Streb LM et al. Genetic linkage of familial open angle glaucoma to chromosome 1q21-q31. Nat Genet 1993; 4: 47-50.

8 Sommer A, Katz J, Quigley HA, Miller NR, Robin AL, Richter RC et al. Clinically detectable nerve fibre layer atrophy precedes the onset of glaucomatous field loss. Arch Ophthalmol 1991; 109: 77-83.

9 Quigley HA, Addicks EM, Green RW. Optic nerve damage in human glaucoma. Quantitative correlation of nerve fibre loss and visual field defect in glaucoma, ischaemic neuropathy, papilloedema and toxic neuropathy. Arch Ophthalmol 1982; 100: 135-146.

10 Quigley HA, Dunkelberger GR, Green WR. Retinal ganglion cell atrophy correlated with automated perimetry in human eyes with glaucoma. Am J Ophthalmol 1989; 107: 453-464.

11 Quigley HA. Examination of the retinal nerve fiber layer in the recognition of early glaucomatous damage. Trans Am Ophthalmol Soc 1986; 84: 920-966.

12 Honrubia FM, Calonge B. Evaluation of the nerve fiber layer and peripapillary atrophy in ocular hypertension. Int Ophthalmol 1989; 13: 57-62.

13 Weinreb RN, Shakiba S, Zangwill L. Scanning laser polarimetry to measure the nerve fibre layer of normal and glaucomatous eyes. Am J Ophthalmol 1995; 119: 627-636.

14 Schuman JS, Pedut-Kloizman T, Herzmark E, Hee MR, Wilkins JR, Coker JG et al. Reproducibility of nerve fibre layer thickness measurements using optical coherence tomography. Ophthalmology 1996; 103: 1889-1898.

15 Sanchez-Galeana C, Bowd C, Blumenthal EZ, Gokhale PA, Zangwill LM, Weinreb RN. Using optical imaging summary data to detect glaucoma. Ophthalmology 2001; 108: 1812-1818. 
16 Bowd C, Zangwill LM, Berry CC, Blumenthal EZ, Vasile C, Sanchez-Galeana $\mathrm{C}$ et al. Detecting early glaucoma by assessment of retinal nerve fibre layer thickness and visual function. Invest Ophthalmol Vis Sci 2001; 42: 1993-2003.

17 Zangwill LM, Bowd C, Berry CC, Williams J, Blumenthal EZ, Sánchez-Galeana CA et al. Discriminating between normal and glaucomatous eyes using the Heidelberg retina tomograph, GDx nerve fibre analyzer and optical coherence tomograph. Arch Ophthalmol 2001; 119: 985-993.

18 Chang YS, Sohn YH. The relationship between optical coherence tomography and scanning laser polarimetry measurements in glaucoma. Korean J Ophthalmol 2006; 20: 225-229.

19 Greaney MJ, Hoffman DC, Garway-Heath DF, Nakla M, Coleman AL, Caprioli J. Comparison of optic nerve imaging methods to distinguish normal eyes from those with glaucoma. Invest Ophthalmol Vis Sci 2002; 43: 140-145.

20 Bagga H, Greenfield DS, Feuer W, Knighton RW. scanning laser polarimetry with variable corneal compensation and optical coherence tomography in normal and glaucomatous eyes. Am J Ophthalmol 2003; 135: 521-529.

21 Leung CK, Chan W, Chong KKL, Yung WH, Tang KT, Woo $\mathrm{J}$ et al. comparative study of retinal nerve fiber layer measurement by stratus OCT and GDx VCC, I: correlation analysis in glaucoma. Invest Ophthalmol Vis Sci 2005; 46: 3214-3220.

22 Kanamori A, Nakamura M, Escano MFT, Seya R, Maeda H, Negi A. Evaluation of the glaucomatous damage on retinal nerve fiber layer thickness measured by optical coherence tomography. Am J Ophthalmol 2003; 135: 513-520.

23 Rhee DJ, Greenfield DS, Chen PP, Schiffman J. Reproducibility of retinal nerve fiber layer thickness measurements using scanning laser polarimetry in pseudophakic eyes. Ophthalmic Surg Lasers 2002; 33: 117-122.

24 Reus NJ, Lemij HG. Diagnostic accuracy of the GDx VCC for glaucoma. Ophthalmology 2004; 111: 1860-1865.

25 Mabuchi F, Aihara M, Mackey MR, Lindsey JD, Weinreb RN. Regional optic nerve damage in experimental mouse glaucoma. Invest Ophthalmol Vis Sci 2004; 45: 4352-4358.

26 Nouri-Mahdavi K, Hoffman D, Tannenbaum DP, Law SK, Caprioli J. Identifying early glaucoma with optical coherence tomography. Am J Ophthalmol 2004; 137: 228-235.

27 Parikh RS, Parikh S, Sekhar GC, Kumar RS, Prabakaran S, Babu JG et al. Diagnostic capability of optical coherence tomography (Stratus OCT 3) in early glaucoma. Ophthalmology 2007; 114: 2238-2243. 